\title{
Dialisi peritoneale: uno sguardo rivolto al futuro
}

\author{
Pasquale Esposito $^{1}$, Elisa Buonanno ${ }^{2}$, Giuseppe Castellano ${ }^{3}$, Carlo Jovane ${ }^{4}$, Daniela Pogliani ${ }^{4}$, \\ Silverio Rotondi ${ }^{5}$, Gianluigi $\mathrm{Zaza}^{6}$, Yuri Battaglia ${ }^{7}$
}

${ }^{1}$ Unità Operativa di Nefrologia, Dialisi e Trapianto, Fondazione IRCCS Policlinico "San Matteo" e Università di Pavia, Pavia

${ }^{2}$ Internal Medicine, Maasstad Hospital, Rotterdam - the Netherlands

${ }^{3}$ Unità Operativa di Nefrologia, Dialisi e Trapianto, Università degli Studi di Bari, Bari

${ }^{4}$ Unità Operativa di Nefrologia-Dialisi, Azienda Ospedaliera "S. Antonio Abate", Gallarate (VA)

${ }^{5}$ Unità Operativa di Nefrologia e Dialisi, Azienda Policlinico Umberto I, Università "Sapienza”, Roma

${ }^{6}$ Unità Operativa Complessa di Nefrologia e Dialisi, Azienda Ospedaliero-Universitaria Integrata di Verona, Verona

${ }^{7}$ Unità Operativa Complessa di Nefrologia e Dialisi, Azienda Ospedaliero-Universitaria "Sant' Anna", Ferrara

\begin{abstract}
Peritoneal dialysis: a LoOK AT THE FUture
Abstract. Several factors may be responsible for the insufficient diffusion of peritoneal dialysis (PD) in Italy, including the lack of cultural understanding, organizational problems, and inappropriate health policies.

Therefore, different approaches have been proposed to face these issues leading to a wider use of this technique.

Among these strategies we think that a key role should be played by the promotion of educational initiatives mostly addressed to young nephrologists. At the same time, development of new technologies, such as domotics and web-based applications, might help to make PD more acceptable and suitable for both patients and physicians.

These are some of the most important challenges to promote PD growth in the future.
\end{abstract}

Key words: Peritoneal dialysis, Domotics, Educational initiatives

Conflict of interest: None.

Financial support: None.

Accettato: 10 Ottobre 2013

Nonostante le evidenze di efficacia e buona accettazione da parte dei pazienti, la dialisi peritoneale (DP) incontra ancora notevoli difficoltà di diffusione nella pratica clinica quotidiana.

Come evidenziato dai precedenti interventi, le ragioni alla base di questa "marginalizzazione" della DP sono molteplici, includendo motivazioni di origine culturale e organizzativa e di politica sanitaria.

Le strategie che possono essere messe in campo prendono in considerazione tutte queste questioni e debbono necessariamente coinvolgere tutti gli attori in campo: i pazienti, il personale sanitario (medici e infermieri) e gli amministratori.

Un approccio complementare e, in qualche modo, "alternativo", a nostro avviso, potrebbe basarsi sull'innovazione. L'introduzione di nuove indicazioni, strumentazioni e metodiche potrebbe, infatti, contribuire al superamento delle barriere che ancora oggi si frappongono a una diffusione adeguata della DP nella popolazione dialitica italiana.

Lo sviluppo di un tale approccio è stato anche uno dei punti fondamentali della recente "Accademia delle Idee", un'iniziativa promossa dalla Società Italiana di Nefrologia per offrire ai giovani una vetrina per la presentazione di progetti innova- tivi in diversi ambiti della pratica nefrologica (1).

Avendo anche alle spalle questa esperienza, ci sembra di poter individuare alcuni ambiti in cui l'investimento nella formazione dei giovani e nell'introduzione di processi e tecniche innovativi possa "demarginalizzare" la DP.

\section{Investire sulla formazione dei giovani}

Sono sempre più diffuse un'esigenza e una domanda di formazione crescenti, affinché la Nefrologia in generale e la DP in particolare si possano riappropriare delle proprie competenze. Rendere autonomi i giovani nella gestione complessiva del paziente in DP, dalla preparazione del peritoneo fino alla prescrizione della terapia e al follow-up del paziente, potrebbe aiutare la DP ad avere una diffusione maggiore anche nei centri più piccoli e periferici.

\section{Nuove indicazioni della DP}

Le evidenze di un uso "non convenzionale" della DP sono sempre più numerose. In particolare, il trattamento dello scompenso 
cardiaco sinistro e destro e delle cirrosi cardiogene appare come un campo di sviluppo molto promettente per la DP (2).

Lo studio e la validazione di algoritmi terapeutici che inseriscano la DP nel percorso di cura del paziente cardiopatico scompensato, insieme ai presidi più tradizionali, come i diuretici e l'ultrafiltrazione dialitica, potrebbero ampliare la conoscenza e la diffusione della DP.

\section{Miglioramento della gestione domiciliare della DP}

L'utilizzo ormai ampiamente diffuso, nelle diverse fasce della popolazione, di Internet, smartphone e tablet apre nuove prospettive per le terapie domiciliari e per la DP in particolare. Lo sviluppo della telemedicina e della domotica potrebbe aiutare, da un lato, a rendere più user-friendly la gestione della DP da parte del paziente e, dall'altro, permettere un migliore monitoraggio clinico da parte del personale sanitario. Esempi di eventuali sviluppi tecnologici in DP, in parte già messi in pratica in alcune limitate esperienze, potrebbero essere l'introduzione di sistemi di comunicazione web-based tra domicilio del paziente e ospedale, l'utilizzo di biosensori che possano aiutare il paziente e gli operatori sanitari a monitorare in tempo reale i parametri clinici principali (peso, stato di idratazione, ecc.) e lo sviluppo di applicazioni per cellulari che assistano il paziente nei diversi aspetti della propria cura (p. es., gestione della terapia farmacologica, informazione sulla composizione degli alimenti, ecc.).

\section{Migliore e più precoce gestione delle complicanze}

Le complicanze della DP e, in particolare, la peritonite sono state per diverso tempo un ostacolo alla diffusione di questa tecnica.

L'introduzione di nuovi device per la connessione sicura del paziente e di nuove tipologie di catetere e l'uso di nuovi protocolli di medicazione hanno sicuramente contribuito a ridurre il tasso di infezioni e di ospedalizzazione (3).

La diagnosi di peritonite resta, comunque, un momento fondamentale, anche perché detta la tempistica dell'intervento terapeutico e può condizionarne l'esito.

Tale diagnosi si basa, però, ancora, sostanzialmente sulla clinica (sintomatologia, dialisato torbido) e può essere ritardata dalla poca consapevolezza del paziente.

L'introduzione di sistemi di diagnosi precoce di peritonite potrebbe aiutare nella gestione di questa temibile complicanza.

L'uso di sistemi turbidimetrici per l'analisi in tempo reale del dialisato o di sistemi biochimici per la detezione delle cellule e dei marcatori infiammatori è una delle potenziali innovazioni che potrebbero permettere una diagnosi più precoce della peritonite in DP.

\section{Aumento della durata della tecnica}

Un limite intrinseco della DP è la sua durata limitata, dovuta alle alterazioni strutturali del peritoneo nel corso del tempo. Lo studio e l'analisi dei fattori legati all'alterazione del peritoneo e alla fibrosi potrebbero, teoricamente, aprire la strada a terapie volte a preservare la membrana peritoneale, permettendo, quindi, una maggiore durata della DP stessa (4).

Questi sono, a nostro avviso, alcuni dei campi di ricerca e di applicazione clinica che potranno interessare la DP nel prossimo futuro.
Non bisogna tralasciare il fatto che lo sviluppo di questi ambiti di ricerca potrebbe aiutare anche a ridurre i costi di questa metodica, aspetto che assume un'importanza sempre più rilevante nella pianificazione della Sanità nel nostro Paese.

In un tempo critico come il nostro, può risultare difficile parlare di innovazione, sviluppo e investimento, ma noi vogliamo aprire anche questa finestra, convinti che si può andare avanti e migliorare analizzando il presente con lo sguardo rivolto al futuro.

\section{Riassunto}

Molti fattori possono condizionare la scarsa diffusione della dialisi peritoneale (DP) in Italia, tra cui deficit culturali, problemi organizzativi e politiche sanitarie inadeguate.

Per ovviare a tali ostacoli sono state proposte diverse strategie di intervento.

Noi riteniamo che la promozione di iniziative educative rivolte, in particolare, ai giovani così come lo sviluppo di nuove tecnologie e metodiche possano rivestire un ruolo chiave per promuovere la DP, rendendola più accessibile sia da parte dei pazienti che dei medici stessi.

Questa, a nostro avviso, resta una delle sfide maggiori per la futura crescita della DP in Italia.

Parole chiave: Dialisi peritoneale, Domotica, Iniziative educazionali

Dichiarazione di conflitto di interessi: Gli Autori dichiarano di non avere conflitto di interessi.

Contributi economici degli autori: Gli Autori dichiarano di non aver ricevuto sponsorizzazioni economiche per la preparazione dell'articolo.

Indirizzo degli Autori:

Dr. Yuri Battaglia

U.O.C. di Nefrologia e Dialisi

A.O.U. Arispedale "Sant'Anna"

Via Aldo Moro 8

44124 Cona (FE)

battagliayuri@gmail.com

\section{Bibliografia}

1. Bellasi A, Becherucci F, Bruno S, et al. L'accademia delle idee. Giornale Italiano di Nefrologia 2013; 30 (2).

2. Rizkallah J, Sood MM, Reslerova M, et al. Reduced hospitalizations in severe, refractory congestive heart failure with peritoneal dialysis: a consecutive case series. Clin Nephrol 2013; 80 (5): 334-41.

3. Lim CT, Wong KS, Foo MW. The impact of topical mupirocin on peritoneal dialysis infection rates in Singapore General Hospital. Nephrol Dial Transplant 2005; 20 (8): 1702-6.

4. Lopes Barreto D, Coester AM, Struijk DG, Krediet RT. Can effluent matrix metalloproteinase 2 and plasminogen activator inhibitor 1 be used as biomarkers of peritoneal membrane alterations in peritoneal dialysis patients? Perit Dial Int 2013; 33(5): 529-37. 